\title{
ELECTROMAGNETIC PHYSICS AT NAL
}

R. Wilson

Harvard University

July 18,1968

This report attempts to represent the views of a study made by Harvard University physicists A. Brenner, E. Engels, F. Pipkin, M. Tannenbaum, K. Strauch and T. Walker. Though written at the start of the 1968 NAL Summer Study it should be considered as representative input data rather than conclusions. Even though it is written as if the writer were anxious to do each experiment on successive days next week, it should not be considered an attempt to preempt the field. Rather it represents a list of possible experiments to any one of which the above-named Harvard physicists would be proud to contribute if the opportunity arises. If others are interested also, so much the better.

Introduction

We shall here list the experiments which we believe can "easily" be performed at NAL and at the same time have a great interest. We shall then proceed to a discussion of methods of performing these experiments; two separate facilities are proposed, each for a part of the overall program. One is a $\mu$ beam; the second is a tagged photon beam.

These ideas are not very original. In particular we refer to reports by C. A. Heusch, W. Selove, and Wong and Wilson in $200 \mathrm{BeV}$ Studies in Experimental Use, Vol. III, UCRL-16830. The purpose of 
this report is to be more specific in order that the whole plan be incor porated in some way into experimental-area design.

\section{Experiments}

1. $\sigma_{\text {total }}(\mathrm{\gamma p})$

2. $\sigma_{\text {compton }}(\gamma \mathrm{p})$ at $0<\mathrm{t}<0.2(\mathrm{BeV} / \mathrm{c})^{2}$

3. $\sigma(\gamma \rightarrow \rho)$

$(\gamma \rightarrow \omega) \quad$ Vector meson

$(\gamma \rightarrow \phi) \quad$ photoproduction

4. $\left(\sigma_{\mathrm{A}}-\sigma_{\mathrm{P}}\right)(\gamma \mathrm{p})$

Difference of cross sections $\sigma_{\text {tot }}(\gamma p)$ for helicities antiparallel and parallel.

5. Separation of the nucleon form factors $G_{E p}\left(q^{2}\right)$ and $G_{M p}\left(q^{2}\right)$ at $\mathrm{q}^{2} \simeq 4$ to $7(\mathrm{BeV} / \mathrm{c})^{2}$ using a polarized beam and polarized target.

6. More complex processes:

a) Search for other bosons

b) Virtual photoproduction at moderate energies and low $\mathrm{q}^{2}$

c) Muon production at high excitation energies and high $\mathrm{q}^{2}$

7. Experiments topically interesting but likely, in our opinion, to be come superseded by the time NAL is built. These are included for completeness and to forestall criticism.

a) Wide-angle electron (or $\mathrm{mu}$ ) pairs

8. Other experiments which, in our view, are sufficiently hard that they ought not to be considered at NAL, or else equivalent information can be obtained elsewhere. 


\section{a) High-momentum transfer ep scattering}

9. Experiments discussed by others.

$\mu^{ \pm}$e scattering

$\mu$ bremsstrahlung

$\mu$ tridents

experiments with electrons as a target

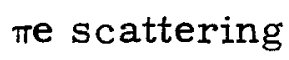

Ke scattering

$\mathrm{K}_{\mathrm{L}}-\mathrm{K}_{\mathrm{S}}$ regeneration from an electron

The reason for the interest in 1, 2, and 4 is the basic study of the forward Compton scattering. We presently expect $\sigma_{\text {tot }}(\gamma p)$ to stay roughly constant at $100 \mu \mathrm{b}$, as the $\gamma$-ray energy increases above $6 \mathrm{BeV}$. But this is a prediction based on vector meson dominance which relates $\sigma_{t}(\gamma p)$ to $\sigma_{t}(\rho p)$ and hence to the constant cross sections of hadron physics. Vector dominance models have some problems. Therefore, this must be checked. The constancy of

$$
\sigma_{\text {tot }}(\mathrm{k}) \quad\left[=\operatorname{Im} \sigma_{\text {compton }}(\mathrm{k}) \text { at } \mathrm{t}=0\right] \text {, }
$$

as a function of energy implies a small real part of $\sigma_{\text {compton }}$ Indeed the dispersion relation tells us that a study of $R e \sigma_{\text {compton }}(t=0)$ may be a more sensitive test of the constancy of $\sigma_{\text {tot }}$ than a study of $\sigma_{\text {tot }}$ itself. This is one way of understanding the experiments of Lindenbaum's group on $\pi p$ scattering. Thus, these experiments are similar in purpose to $\pi p$ scattering but for a simpler particle (the $\gamma$ ray). 
The forward compton scattering tells us $|\operatorname{Im} f(0)|^{2}+|\operatorname{Re} f(0)|^{2}+$ + spin terms whence we find Re $f(o)$. The $t$ dependence can and will be compared with the $t$ dependence of $\gamma \rightarrow \rho$ and $\gamma \rightarrow \omega$ experiments and the $t$ dependence of hadronic scattering cross sections as a check on the combination of $\mathrm{SU} 3$ and vector dominance. We expect on this basis something close to $\exp (10 t)$.

The study of experiment 4 is of the spin dependence of the compton cross section. This has recently attracted the attention of theorists who believe that the spin-dependent part of the cross section vanishes, as the energy increases, faster than the nonspin-dependent part. The direct experiment suggested here has not been done at any energy, and the vanishing at high energies is inferred from the Drell-HearnGerazimov sum rule, and the spin-dependent cross sections in the sum rule are obtained from a detailed analysis of photoproduction data in the region of the 1238,1512 , and 1688 nucleon resonances. The direct experiment, if possible, and we think it is, is clearly exciting.

The experiment 3 is a check on vector dominance predictions; the cross section for these processes in the $4-\mathrm{BeV}$ region is well predicted by a knowledge of the $\gamma \rho$ coupling and the $\rho$ nucleon cross sections. Does this persist at high energies? And, in particular, is the $\gamma \rho / \gamma \phi$ ratio in agreement with theory?

The experiment 5 is hard to do at SLAC. It involves comparing forward (measuring $G_{E}{ }^{2}+q^{2} / 4 M^{2} G_{M}{ }^{2}$ ) and backward (measuring $G_{M}{ }^{2}$ ) 
ep scattering and pulling out, thereby the small term $\left[G_{e p}\left(q^{2}\right)\right]$ which in the region stated, probably contributes from 5 to $10 \%$ of the forward cross section. By using a polarized lepton beam (mu beam) and polarized target, we find a term $\pm G_{E} G_{M}{ }^{*}$ dependent on the relative sign of the lepton helicity and the target polarization. We can hope that this term can be found relatively free of systematic errors. Of course the counting rate will not compare with that at SLAC so that this is the only lepton proton scattering experiment proposed as a measure of nucleon form factors. Other $\mu$ p scattering experiments can study the $\mu \gamma$ vertex.

We shall postpone discussion of the harder experiments until later. The experiments proposed for the tagged photon beam are 1, 2, and 3. Those for the muon beam are 1, 4, and possibly 3, also. We shall now proceed to discuss the experimental design for each of them.

\section{Tagged $\gamma$-Ray Beam}

The design of a tagged $\gamma$-ray beam suitable for energies up to 100 $\mathrm{BeV}$ has been discussed by Heusch in the 200-BeV Study Report already noted (UCRL-16830, p. 156). We have gone through these calculations and confirm them to a usual factor of 2 or so. There are two proposed methods for obtaining an electron beam, Figs. 8(b) and 9 . In the first, electrons from the target (in the forward direction of course) come from $\pi^{\circ}$ decay and subsequent conversion of the $\gamma$ rays. These electrons pass *See, for example, a forthcoming paper by Norman Dombey. 
through a transport system which has an unusually large $\Delta p / p=0.1$ but low resolution (1\%). At the end of this system we anticipate the ratio $\mathrm{e}^{-} / \pi^{-} / \overline{\mathrm{pK}}=1 / 20 / 22$. The second system takes the neutral beam; $y$ rays are converted by one radiation length of lead and again there is a transport system. At the end of the beam we expect $e^{-} / \pi^{-}$to be $1 / 10^{-2}$ or less. Thus, the second system is preferable; it remains, however, to see which system fits in with overall NAL planning. A third system of W. Toner at SLAC has not been considered.

We now detail the second system a little more. Such a beam transport was discussed by Longo, UCID-10123, Vol. 2, p. 23.

We note that the intensity of the tagged $\gamma$-ray beam is equal to that of the beam at CEA set up by Strauch, Walker, and Brenner. The duty cycle of the accelerator is 10 times better. Thus, before we proceed, we can make the general statement that anything that can be done at CEA can be done at NAL. Some detail on the tagged gamma experiments will follow, but fuller details can be found in proposals to CEA by Caldwell, Frisch, Strauch, Walker, and Brenner.

\section{Total Cross Section}

This experiment has been proposed many times at synchrotrons and never yet successfully performed. At Cornell, Berkelman et al. ran into various problems at $\mathrm{E}_{Y}=2 \mathrm{GeV}$ and abandoned the experiment. It is our belief that the experiment is easier at high energies.

We shall consider an apparatus which measures $\sigma_{\text {total }}$ by 2 methods 
simultaneously. The first, an attenuation method, and the second by interpreting all differential cross sections. These would be easy experiments except that we are trying to measure a total nuclear cross section of $100 \mu$ barns in the presence of a 30 millibarn pair production cross section.

The ideas which make the absorption experiment possible are twofold. First, the use of a tagged $\gamma$ beam and second, the suggestion made to $R$. Wilson in 1961 by $H$. Joos, that a good shower counter has the same response to an electron positron pair as to the $\gamma$ ray which produced the pair. The electron which tags the gamma ray gives an indication that a gamma ray is emitted; if a $\gamma$ ray arrives for $100 \%$ of the electron signals, we can work with a low attenuation in the target without bad loss of statistical accuracy. The detector is a shower counter and only the nuclear processes in the target lead to absorption. Before going into some problems, we shall note the second method of detection to be performed simultaneously; the hydrogen target is to be surrounded by an arrangement of counters, with some lead to convert $\pi^{\circ}$ decay $\gamma$ rays, covering nearly $4 \pi$; the forward angle is excluded. The total cross section is defined by a $\gamma$ ray giving a count in one of the hodoscopes. Combining both methods, each time there is a count in the hodoscope, there should be no count in the shower counter and vice versa; spurious effects may be studied by a detailed examination of hodoscope counts. We note here that W. Toner of SLAC stresses the 
advantage of using a short hydrogen target in the second method to re duce second order $\left(t^{2}\right)$ electromagnetic effects. Clearly there is no advantage in reducing the target thickness below

$$
t=2 \alpha / \pi \log (\mathrm{E} / \mathrm{m}) \approx 0.02 \text { radiation lengths. }
$$

\section{Total Cross-Section Detailed Problems}

(a) Inefficiency of Tagging

In every tagging system built so far (DESY, CEA, Cornell), there does not exist one high-energy $\gamma$ ray for every low-energy electron. This efficiency is typically $92 \%$. It is very important to make this num ber high in order that a low attenuation in the target does not degenerate the statistical error for the absorption method. Although it is not certain that all the causes of the inefficiency have not been included below, it is hoped that many are. Some electrons may strike magnet walls and produce $\gamma$ rays in the wrong direction. We obviate this by making the radiator a scintillator and putting it in coincidence. Some electrons radiate twice. This varies roughly as $(\log E / m+t / 2)$, where $t$ is the thickness of the target in radiation lengths. We reduce $t$, for this experiment at least, to 0.01 so that the second term is less than the first. The loss of intensity is no problem because the experiment will almost certainly be limited by systematic errors. Electron tridents can be produced in the radiator--again roughly proportional to $(\log \mathrm{E} / \mathrm{m}+\mathrm{t} / 2)$, and these are further reduced by an anticoincidence on any produced 
positron. An efficiency of $98-99 \%$ should be achieved, but one of $95 \%$ is almost guaranteed from present experience. To cope with the residual inefficiency we shall of course measure a hydrogen in/out effect. The count rate for the total cross section will be (a bar represents an anticoincidence)

$$
\mathrm{C}_{1} \mathrm{C}_{2} \mathrm{C}_{3} \overline{\mathrm{C}}_{4} \overline{\mathrm{C}}_{5} \overline{\mathrm{C}}_{7}\left(\mathrm{H}_{2} \text { in }\right)-\mathrm{C}_{1} \mathrm{C}_{2} \mathrm{C}_{3} \overline{\mathrm{C}}_{4} \overline{\mathrm{C}}_{5} \overline{\mathrm{C}}_{7}\left(\mathrm{H}_{2} \text { out }\right)
$$

for the attenuation method. We can reach a maximum tagged $\gamma$ rate of about $10^{5} / \mathrm{sec}$, reduced from $10^{6} / \mathrm{sec}$ by use of a 0.01 radiation length radiator instead of 0.1 radiation lengths. This is the coincidence $\left(\mathrm{C}_{1} \mathrm{C}_{2} \mathrm{C}_{3} \overline{\mathrm{C}}_{4} \overline{\mathrm{C}}_{5}\right)$. With an efficiency of $98 \%$ we get a rate of $2 \times 10^{3} / \mathrm{sec}$ for $\mathrm{C}_{1} \mathrm{C}_{2} \mathrm{C}_{3} \overline{\mathrm{C}}_{4} \overline{\mathrm{C}}_{5} \overline{\mathrm{C}}_{7}\left(\mathrm{H}_{2}\right.$ out) and with a 2 meter $\mathrm{H}_{2}$ target we have an absorption of $8 \times 10^{-4}$. This extra contribution to $\mathrm{C}_{1} \mathrm{C}_{2} \mathrm{C}_{3} \overline{\mathrm{C}}_{4} \overline{\mathrm{C}}_{5} \overline{\mathrm{C}}_{7}$ is then $80 / \mathrm{sec}$, to be found in the presence of the target out effect of $2 \times 10^{-3}$. The importance of keeping the efficiency high--and the target out rate low--is then obvious. One day is $\sim 10^{5} \mathrm{sec}$, we get $\sim 2 \times 10^{8}$ counts with target out and an extra $6 \times 10^{6}$ counts with target in, found to an error of $1.4 \sqrt{2 \times 10^{8}}=2 \times 10^{4}$. Thus a statistical error of $3 \times 10^{-3}$ in the actual cross section is easy to obtain. Of course this must be spread over several bins of $\mathrm{E}_{\gamma}$. Systematic errors vary moderately with $E_{\gamma}$; thus, an aim of a $1 \%$ measurement (relative) of $\sigma_{\text {total }}$ in each of 100 bins can be achieved in 4 days. Bumps in $\sigma_{\text {tot }}$ can then be found if they exist. We note that it is bumps in $\sigma_{\text {tot }}$ vs energy which contribute to $\operatorname{Re} f(0)$ through the dispersion relation. 
For an absorption of $8 \times 10^{-4}$ due to the strong interactions there is an absorption due to pair production of $30 \%$ and secondary processes of $10 \%$. Secondary processes are hard to calculate; some of them will produce electrons--or worse still protons or pi's--at large angles. Provided, however, that electrons and gamma rays proceed forward, the sum of whose energies exceeds, say, E/10, there should be no problem. Of course, this is a place for twelve months' detailed work by the graduate student before the experiment starts. Two checks on this are available. We can replace the target by a shorter length (to reduce $\mathrm{t}^{2}$ effects), and we can use a spread out high $Z$ target (uranium) of the same pair productions $(30 \%)$ where the total cross -section measurement should be down by $238 \times 2 / 92^{2}=4 \times 10^{-2}$. The 2 comes from $Z(Z+1)$ for hy drogen. If it is not, spurious effects remain. The other check is the examination of the counters $\mathrm{C}_{6}$ when an anticoincidence occurs. We expect a few large-angle electrons, positrons, $\gamma$ rays on the trigger $\mathrm{C}_{1} \mathrm{C}_{2} \mathrm{C}_{3} \overline{\mathrm{C}}_{4} \overline{\mathrm{C}}_{5} \overline{\mathrm{C}}_{7}$. These should be reached forward (and in agreement with the hypothetical graduate student's calculations). If too many, there is trouble.

The other way of measuring the cross section uses the trigger $\mathrm{C}_{1} \mathrm{C}_{2} \mathrm{C}_{3} \overline{\mathrm{C}}_{4} \overline{\mathrm{C}}_{5} \mathrm{C}_{6}\left(\mathrm{H}_{2}\right.$ in $-\mathrm{H}_{2}$ out $)$, again we expect this to correspond to $\overline{\mathrm{C}}_{7}$. The counts which are uncertain are again those which give $\mathrm{C}_{1} \mathrm{C}_{2} \mathrm{C}_{3} \overline{\mathrm{C}}_{4} \overline{\mathrm{C}}_{5} \mathrm{C}_{6} \mathrm{C}_{7}$. For $10^{5} / \mathrm{sec}$ tagging electrons $\left(\mathrm{C}_{1} \mathrm{C}_{2} \mathrm{C}_{3} \overline{\mathrm{C}}_{4} \overline{\mathrm{C}}_{5}\right)$ we expect $80 / \mathrm{sec}, \mathrm{C}_{1} \mathrm{C}_{2} \mathrm{C}_{3} \overline{\mathrm{C}}_{4} \overline{\mathrm{C}}_{5} \mathrm{C}_{6}\left(\mathrm{H}_{2}\right.$ in) and nothing with $\mathrm{H}_{2}$ out. In one 
day we get $5 \times 10^{6}$ counts with a statistical accuracy of $5 \times 10^{-4}$. For more details of this experiment, see various CEA memos and proposals on the subject of a measurement $\mathrm{E}_{\gamma}=6 \mathrm{BeV}$. We summarize that the problem is to separate the total nuclear cross section from electromagnetic effects 300 times greater. The two methods proposed are complementary with different problems of separation and should be performed simultaneously. Although we have written them as "easy" experiments, we should note problems. In the tagging system it appears, at first sight, that a tagging electron without a tagged gamma ray can only occur by a nuclear interaction. Using the Weiszacker-Williams arguments, we then have a third way of measuring the total nuclear cross section. On this basis, the existing tagged beams give total cross sections 10 times too big. Unless this is understood, and it isn't yet, it would be hard to believe either total cross-measurement. Forward Compton Scattering

There are two parts to this experiment, separated by the region of momentum transfer $(-t)$. If the recoil proton is detected, $(-t)$ is limited to being greater than 0.1 or $0.2(\mathrm{BeV} / \mathrm{c})^{2}$. " Since the cross section is expected to vary as $e^{10 t},(-t)=0.1$ is not a small angle. Thus, I shall assume that the proton is not detected. Another experiment, not here discussed, (but probably not with a tagged $\gamma$-ray beam for intensity reasons) can be done for $(-t)>0.1$ $(\mathrm{BeV} / \mathrm{c})^{2}$ and detecting the proton. We shall need to measure the *Gas targets and/or bright ideas may reduce this. 
incident $\gamma$-ray energy and the scattered $\gamma$-ray energy; we can then specify the reaction $\gamma+p \rightarrow \gamma+p$.

The backgrounds expected are the inelastic processes $\gamma+p \rightarrow \gamma^{\prime}+\ldots$ (which is probably small) and the forward $\pi^{\circ}$ production accompanied by forward $\pi^{\circ}$ decay. This latter will always produce a $\gamma$ ray equal to the incident $\gamma$ ray less the $\pi^{\circ}$ mass (and any other masses produced). At $\mathrm{E}_{\mathrm{Y}}=4 \mathrm{BeV}$, the $\pi^{\circ}$ production is a large background. A measurement of the scattered $\gamma$-ray energy to $2 \%$ (full-width, half-max. of $4 \%$ ) seems adequate. Now $(\mathrm{d} \sigma / \mathrm{dt})(\mathrm{t}=0)=(1 / 2) \mu \mathrm{b} / \mathrm{GeV}^{2}$ neglecting the real part of the amplitude. $(\mathrm{d} \sigma / \mathrm{dt})_{\mathrm{t}=0}\left(\pi^{\mathrm{O}}\right.$ production $)$ is proportional to $1 / \mathrm{s}$. Thus we expect to be better off.

One possible $\gamma$-ray detector is a set of sodium iodide crystals as a total absorption counter. The resolution (fwhm) is $\sim 1.5 \%$ at $10 \mathrm{BeV}$ and scales to $1 / 2 \%$ at $100 \mathrm{BeV}$. We can make the resolution of the tagging system similar by (a) measuring the incident electron energy to $0.25 \%$; (b) measuring the scattered electron to $0.5 \%$ using a fine enough hodoscope. Here the only problem we see is the radiative correction in the tagging process; the $\gamma$-ray spectrum will not be within the $0.5 \%$ quoted but will have a low energy tail roughly proportional to

$$
[\alpha / \pi \log (E / m)+t / 2]\left[d k /\left(k_{o}-k\right)\right]
$$

where $\mathrm{k}_{\mathrm{o}}$ is the desired $\mathrm{\gamma}$-ray energy. This, being a low energy tail, should not prevent us from seeing high-energy gamma rays in the presence of lower energy gamma rays. Neglecting this radiative 
problem, we have a resolution of $750 \mathrm{MeV}$ in $100 \mathrm{BeV}$, not enough to kinematically exclude simple $\pi^{\circ}$ production at this energy, but enough with the $1 / \mathrm{s}$ factor noted above. Simple $\pi^{\circ}$ production can probably be kinematically excluded up to $\mathrm{E}_{\gamma}=30 \mathrm{BeV}$. Other $\gamma$-ray detectors are possible.

The arrangement is shown in Fig. 3. The target is surrounded by anticounters to ensure that no charged particle escapes [protons for $(-t)<0.1(\mathrm{BeV} / \mathrm{C})^{2}$ will not] or any wide-angle gamma ray escapes. A shower counter in the forward direction is an anticounter which assures that the incident $\gamma$ ray has interacted. A bank of NaI counters covers $1 / 4$ of the azimuth and covers $(-\mathrm{t})$ to $0.1(\mathrm{BeV} / \mathrm{c})^{2}$. These NaI crystals have a hodoscope in front to define the point of entry of the $\gamma$ ray. Pos sibly one simple large NaI crystal could be used, combining the anticoincidence function. Spurious effects--pair production, electron compton effect, are anticoincidenced by this geometry. So to a large extent is multiple $\pi$ production.

Now the rate of incident $\gamma$ rays is about $10^{6} / \mathrm{sec}$ over an interval $\mathrm{k}_{1} / \mathrm{k}_{2}=2.718$. The total expected compton cross section is $\sigma_{\text {tot }}=1 / 20$ $\mu b$ (excluding the real part of the cross section). In a 1-meter target we, therefore, obtain one scatter every 2 seconds of which we select $t \geq 1 / 4$; thus we can hope for 6 counts per minute or 9000 counts per day. If we separate these into 90 bins ( 9 in energy, 10 in t) we still have $10 \%$ statistics in each bin in 1 day. This we regard as a very 
worthwhile experiment. A comparison of Figs. 2 and 3 shows that much of the apparatus is the same. Thus the experiments can be done on successive days (or realistically in successive years).

Photoproduction of Vector Mesons

There is some disagreement even internally in the group of Har vard physicists noted above whether this experiment is best done with real photons or by virtual photons from a muon beam. This disagreement can presumedly be reduced by calculation. As a first step, this section estimates the parameter of the experiment for a photon beam. We assume for calculational purposes:

$$
\begin{aligned}
& \mathrm{d} \sigma / \mathrm{dt}(\gamma \rightarrow \rho)=\left[150 \mu \mathrm{b} \mid(\mathrm{GeV})^{2}\right] \exp (10 \mathrm{t}), \\
& \mathrm{d} \sigma / \mathrm{dt}(\gamma \rightarrow \omega)=\left[20 \mu \mathrm{b} \mid(\mathrm{GeV})^{2}\right] \exp (10 \mathrm{t}), \\
& \mathrm{d} \sigma / \mathrm{dt}(\gamma \rightarrow \phi)=\left[4 \mu \mathrm{b} \mid(\mathrm{GeV})^{2}\right] \exp (10 \mathrm{t}) .
\end{aligned}
$$

We shall detect the $\rho$ by $\rho \rightarrow 2 \pi$ decay; the $\phi$ by $\phi \rightarrow 2 \mathrm{~K}$ decay, the $\omega$ by $\pi^{+} \pi^{-} \pi^{\circ}$ decay.

At $E_{Y}=50 \mathrm{GeV}$, the vector meson takes almost all the forward momentum of the $\gamma$ ray. The transverse momentum is given by the energy release in the decay $p \rightarrow 2 \pi(500 \mathrm{MeV})$. Thus the minimum opening angle is $24 \mathrm{mrad}$ at $50 \mathrm{GeV} / \mathrm{c}$. The production angle for $\approx 0.1 \mathrm{BeV} / \mathrm{c}$ is $2 \mathrm{mrad}$. A magnet system must accept these angles and measure the particle energy to a few percent (to ensure elastic $\rho^{\circ}$ production) and 
opening angle to an accuracy small compared with $\Gamma_{\rho} / \mathrm{M}_{\rho} \approx 1 / 7$ to measure the mass of the $\pi^{ \pm}$pair.

The $\mathrm{K}^{+} \mathrm{K}^{-}$opening angle from $\phi$ decay is much less, due to the low energy release in the decay $\phi \rightarrow \mathrm{K}^{+} \mathrm{K}^{-}(31 \mathrm{MeV})$. At $\mathrm{E}_{\mathrm{\gamma}}=50 \mathrm{GeV}$, we find 5.2 mrad.

One possible system is to use a large $\mathrm{H}$ magnet and measure the track of the pairs by wire spark chambers before and after the magnet. This large $\mathrm{H}$ magnet is preceded by a smaller $\mathrm{H}$ magnet which acts as a clearing field (to clear low energy $\mathrm{e}^{+} \mathrm{e}^{-}$pairs), and also, for the $\phi$ meson to separate the $\mathrm{K}^{+} \mathrm{K}^{-}$a little so that they may be measured.

We shall guard against e pairs by a lack of shower production (again an NaI crystal should be very good; Hofstadter has shown that $\pi$ mesons produce small pulses in such a device).

To separate $\pi^{+} \pi^{-}$( $\rho$ decay $)$ from $\mathrm{K}^{+} \mathrm{K}^{-}(\phi$ decay $)$ is hard. The obvious way is by means of a Cerenkov counter; at low energies this if fine, but at these energies it won't work. Tentatively we suggest not separating and separating the $\rho$ and $\phi$ by measuring the energies only. The $\phi$ will appear, because of the small opening angle, at the low mass end of the $\rho$ peak.

The integrated cross sections, even for $\phi$ production, is about 5 times the compton scattering cross section. We shall, therefore, get $5 \%$ statistics for $\phi$ production in 1 day from hydrogen; we will probably try coherent production off a medium -A material to reduce background. 
The important feature we at once see is the size of the apparatus; the same apparatus is used as for the total cross section and compton cross section plus two magnets downstream. We do not need much width.

\section{Tagged Beam}

The beam we are talking about will be the same for all of these 3 experiments noted above. We note first that the multiple scattering angle of electrons, and the angle of emission of $\gamma$ rays and electrons, is small compared with the accepted angles in the transport system and can be neglected. Therefore, although we start at the target with $\gamma$ rays, convert to electrons in the neutral beam with a radiator, the electrons then radiate to tag, we can consider focusing from accelerator target through to the liquid-hydrogen target, and beyond, to the final shower detector in coincidence for compton scattering and in anticoincidence for the total cross section. This enables us to have a large aperture for the beam without ruining the angular definition of the scattering experiment.

This is illustrated schematically in Fig. 4.

We shall need an intermediate energy focus to enable us to put several counters in an energy defining hodoscope and obtain resolution together with intensity--primarily for the compton experiment.

$\underline{\mu \text { Experiments }}$

We now come to the question of $\mu$ experiments. I refer, as 
background material, to the AGS and SLAC experiments, and to a memo of April 9, 1968, by R. Wilson and comments therein by W. A. Blanpied. The total scattering on hydrogen, integrating over all outgoing directions, tells us $\sigma_{k}$ (total) at a $\gamma$-ray energy $k=E_{1 \text { incident }}-E_{\text {scattered }}$ by Weiszacker-Williams arguments. An integration (trivial but complex), and done already by Liberman, over scattered $\gamma$ energies must be done. A small $(\sim 10 \%$ or so) correction for the inelastic nucleon form factor must be made.

This experiment, with a hydrogen target $30 \mathrm{~cm}$, say, will give 2 events per $10^{6}$ muons. Also present is $\mu$ bremsstrahlung, 4 events $/ 10^{6}$ $\mu^{\prime}$ 's and $\mu$ e scattering, 100 events $/ 10^{6} \mu^{\prime} s$.

The last can be avoided by rejecting coplanarity. For a total cross-section measurement of $3 \%$ we want $500 \times 10^{6}$ muons; a $\mu$ beam of $10^{6} / \mathrm{sec}$ gives the result in a day. $10^{6} / \mathrm{sec}$ is low enough that we can measure the direction and energy of each $\mu$ (as done at the AGS). If higher $\mu$ beams are used, and no trick is found for measuring the energy and direction, the $\mu$ beams must be monochromatic and low angular divergence as at SLAC. (See memo June 1968 by Toner).

This measurement is clearly inferior to the proposed measurement of 1 using a tagged $\gamma$-ray beam. It is mentioned for 3 reasons. First, a $\mu$ beam may be available before the photon beam. Second, it tends to come out automatically from more complex experiments, and third, it is a preliminary to a measure of $\sigma_{\mathrm{A}}-\sigma_{\mathrm{P}}$ now to be mentioned. 


\section{Experiment 4}

The $\mu$ mesons are expected to be $100 \%$ polarized with spin along the direction of motion. This for high energies ( $\mathrm{m} / \mathrm{E}$ small) is an interesting direction. It is just possible to measure the difference of total cross sections for $\mu$ nuclear events, for spins parallel and antiparallel the quantity that Drell-Hearn-Gerazimov have written sum rules about. First, the $\mu$ bremsstrahlung is circularly polarized in the for ward direction. But this is weak and hard to use.

The inelastic $\mu$ scattering can give us our handle on total $\mu$ p cross sections. If the scattering is through an angle $\theta<\mathrm{m} / \mathrm{E}$, the virtual photon is circularly polarized in the same sense as the incident muon spin. If $\theta>\mathrm{m} / \mathrm{E}$, the virtual photon becomes transversely polarized, independent of the initial muon spin. (This is an observation of K. Berkelman, Aug. 1967.) If we take a high-intensity $\mu$ beam, $10^{8} / \mathrm{sec}$, and scatter forward, off a target of $10 \mathrm{~cm}$ alcohol and water, we get at $E_{Y}=50 \mathrm{BeV}$, about 10 scatters per second in the forward direction. The sideways scatters can presumably be avoided by anticoincidence, and in a day we get $10^{6}$ counts. The polarization of the hydrogen in the alcohol can be $30 \%$ in practice (Harvard/CEA 1968) corresponding to $1 / 20$ of the target nucleons being polarized. Thus the statistics of $0.1 \%$ can tell us $\sigma_{A}-\sigma_{P}$ to $0.001 / 0.05=1 / 20$ of the maximum possible.

Improvements in polarized targets can be (i) improvement of polarization in alcohol to $60 \%$; many people are struggling with this, which 
is a cookbook art, (ii) brute force solid hydrogen polarization of $20 \%$. R. V. Pound is working on this. The improvement can be a factor between 2 and 4. Meanwhile, I believe it is a hard experiment, and this note does not claim to have specified a guaranteed way of doing it; the main problems in the way outlined are the handling of the high-intensity $\mu$ beam, avoidance of halos, etc.

We note that the polarized target used here is longitudinal. The usual CERN/Berkeley/Rutherford/Argonne targets have pole tips in the way. However, the Harvard (R. V. Pound) target uses super: superconducting Helmholz coils with the greatest aperture in this direction and so is well suited to this experiment.

Now we come to the other $\mu$ experiment, which also involves a high-intensity $\mu$ beam. This also uses a polarized target. Various authors have calculated lepton-muon scattering for arbitrary polarization over the past 10 years. However, N. Dombey, in a forthcoming paper, asks the useful question, "What is the optimum direction of target polarization?" He finds that with the target polarization perpendicular to the recoil proton in the scattering plane, the cross section attains the simple form

$$
\frac{\mathrm{d} \sigma}{\mathrm{d} \Omega}=\frac{\alpha^{2}}{2 \mathrm{M}^{2}}\left(\frac{\mathrm{E}^{\prime}}{\mathrm{E}}\right)^{2} \frac{1}{1-\epsilon}\left\{\mathrm{G}_{\mathrm{M}}^{2}+\epsilon_{\mathrm{L}} \mathrm{G}_{\mathrm{E}}^{2} \mp 2 \mathrm{G}_{\mathrm{E}} \mathrm{G}_{\mathrm{M}}\left[\epsilon_{L^{(1-\epsilon)}}\right]^{1 / 2}\right\}
$$




$$
\begin{aligned}
& \epsilon_{L}=\frac{4 M^{2}}{q^{2}} \epsilon \\
& \epsilon=\left[\left(1+\epsilon^{2} / 4 M^{2}\right) \tan ^{2} \theta / 2\right]^{-1} .
\end{aligned}
$$

We shall work at small scattering angles so that the curly bracket becomes

$$
\left\{\left(G_{M} \mp G_{E} N \epsilon_{L}\right)^{2}\right\} .
$$

Now at $\mathrm{q}^{2} \approx 4(\mathrm{BeV} / \mathrm{c})^{2}$ the squared term is about $10 \%$ and hard to find; the interference term becomes $\pm 60 \%$ and easy. The effects of low target polarization reduce this. Hopefully, we still get $\pm 30 \%$ with the scattering off the carbon reduced by kinematic definition.

\section{$\underline{\text { Section } 6}$}

We now come to more complex processes.

(a) A search for bosons other than $\rho, \omega, \phi$ can be made, either by real or virtual photon by using missing mass in the reaction $\gamma+p \rightarrow B^{0}+p$. It turns out that the mass resolution (G. Gladding, M. Tannenbaum, and other CEA proposals) is limited by the angle of the proton. This in turn is limited by multiple scattering of the proton in the target. We need a low-energy proton because the cross section varies as

$$
e^{10 t}=e^{-20 M_{p} E_{p}}
$$

Thus we want $E_{p}<100 \mathrm{MV}$ to get the rate. At $E_{p}=6 \mathrm{BeV}$, the resolution 
can be $\pm 3 \mathrm{MeV}$; it gets worse as $\mathrm{E}_{\gamma}$ increases, roughly as $\mathrm{E}_{\gamma}$. At 60 $\mathrm{BeV}, \Delta \mathrm{m} / \mathrm{m}= \pm 30 \mathrm{MeV}$, still interesting if the resonances are not too numerous.

(b) In general the apparatus for the total or compton cross section can be made into a general apparatus by adding streamer chambers (courtesy of A. Odian and others) or other devices around the target and in a magnetic field. We make no comment on wide-angle electron pairs except to refer to the Berkeley report on experimental use, UCRL-16830. Finally, we calculate the obvious $\mu p$ elastic scattering to see if we can exceed the SLAC momentum transfer. SLAC were rate limited (5/day) at $\mathrm{q}^{2}=25(\mathrm{BeV} / \mathrm{c})^{2}$. They used $10^{13} \mathrm{e} / \mathrm{sec}$ and a $10 \mathrm{~cm}$ target, and $100 \mu$ radian solid angle. With $\mu$ mesons we can have $10^{8} \mathrm{e} / \mathrm{sec}$; $100 \mathrm{~cm}$ target and 100 mradian (because the background and duty cycles are less). We are down in rate by a factor of 10 . Thus $\mu$ p elastic scattering can (with the exception of the polarized target experiment) only compare e $\gamma$ and $\mu \gamma$ vertices. Perl and Tannenbaum have independently pointed out the advantage of using inelastic scattering for this, with a higher cross section.

Important experiments omitted from this report include some which are of interest primarily for a study of electron- $\mu$ meson differences and not for electromagnetism- $\mu$ p elastic scattering, $\mu$ bremsstrahlung, $\mu$ e scattering, $\mu$ tridents. 
More serious is an omission of some exciting experiments with electrons as target. Te scattering, Ke scattering, $K_{L} \rightarrow K_{S}$ regeneration from an electron. 


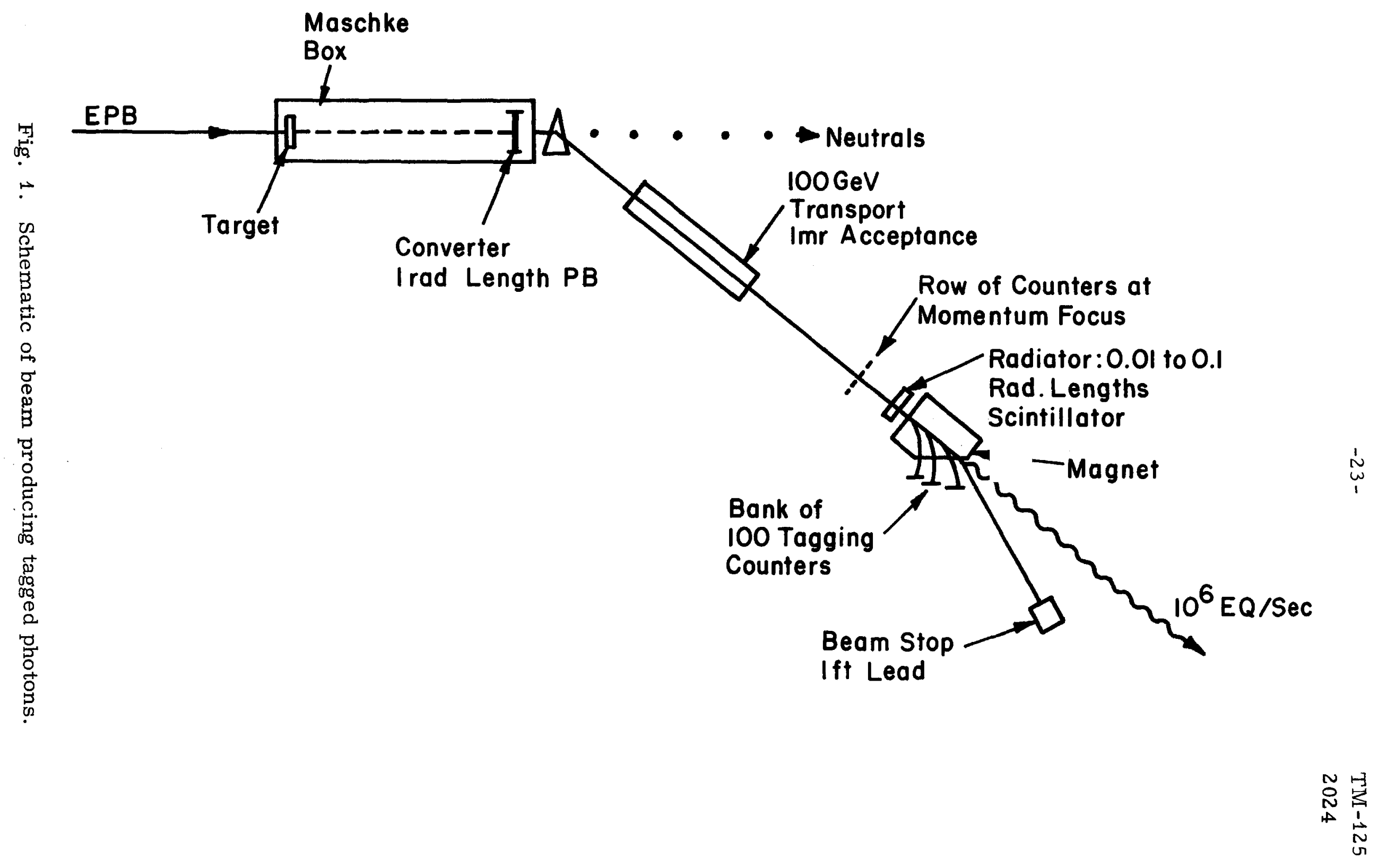




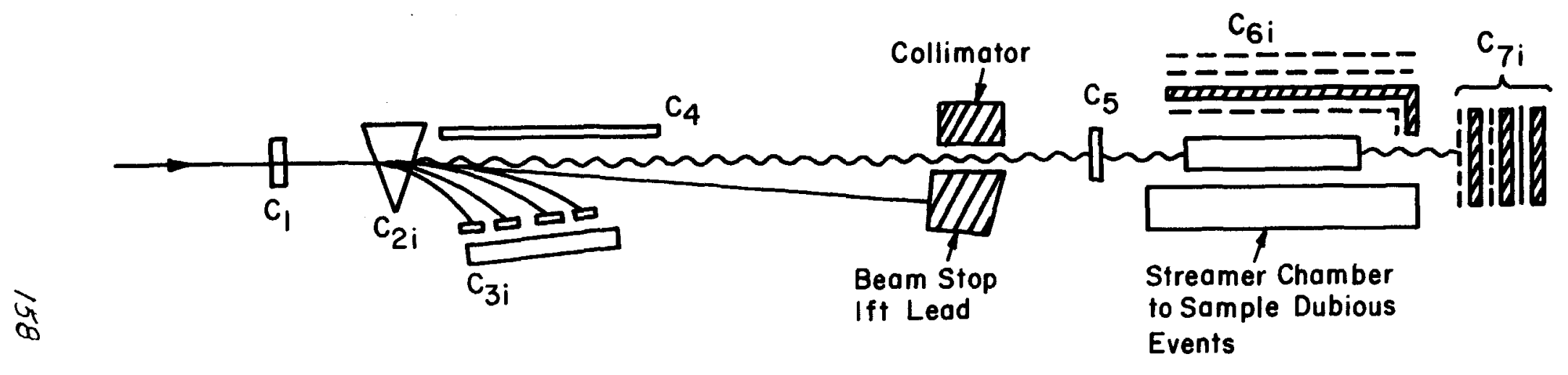

Events

Fig. 2. Total cross-section measurement with tagged photon beam. $C_{1}=0.01$ rad length scintillator, $\mathrm{C}_{2 i}, \mathrm{C}_{3 i}=$ tagging counters, $\mathrm{C}_{4}=$ anticoincidence positron counters, $\mathrm{C}_{5}=$ anticoincidence charged beam counter, $\mathrm{C}_{6 i}=$ event-detecting counters, $\mathrm{C}_{7 i}=$ scintillator-lead sandwich or NaI counters. The hydrogen target is just above the streamer chamber. 


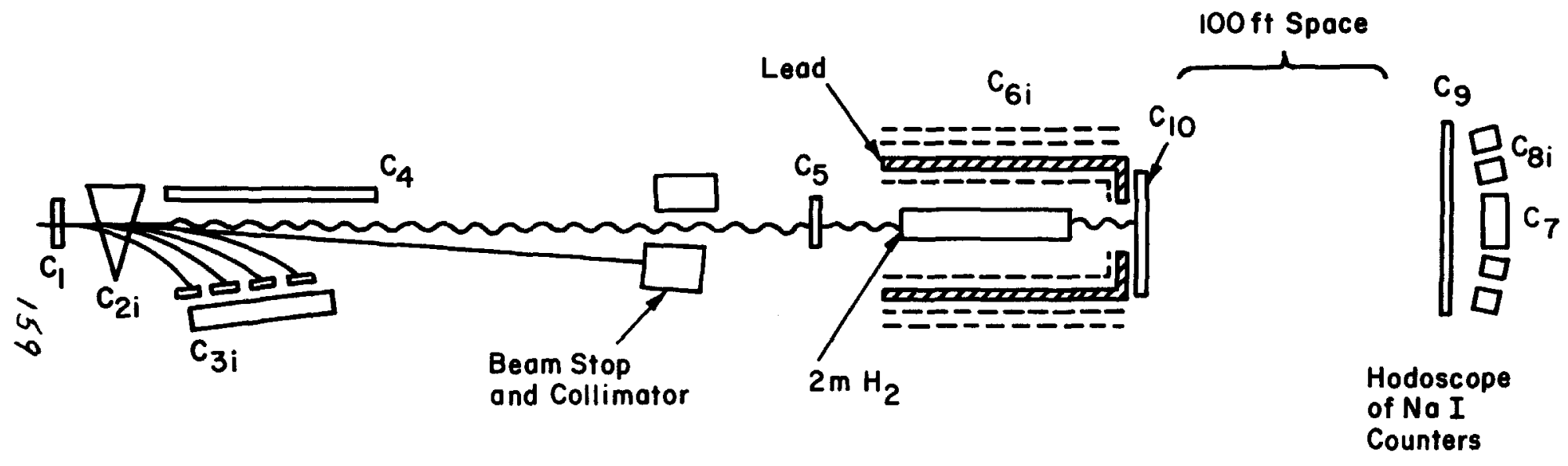

Fig. 3. Experiment on forward Compton scattering. $C_{1}, C_{2 i}, C_{3 i}$,

$\mathrm{C}_{8 \mathrm{i}}=$ coincidence counter $\mathrm{s}, \mathrm{C}_{4}, \mathrm{C}_{5}, \mathrm{C}_{6 \mathrm{i}}, \mathrm{C}_{7}, \mathrm{C}_{9}, \mathrm{C}_{10}$ 

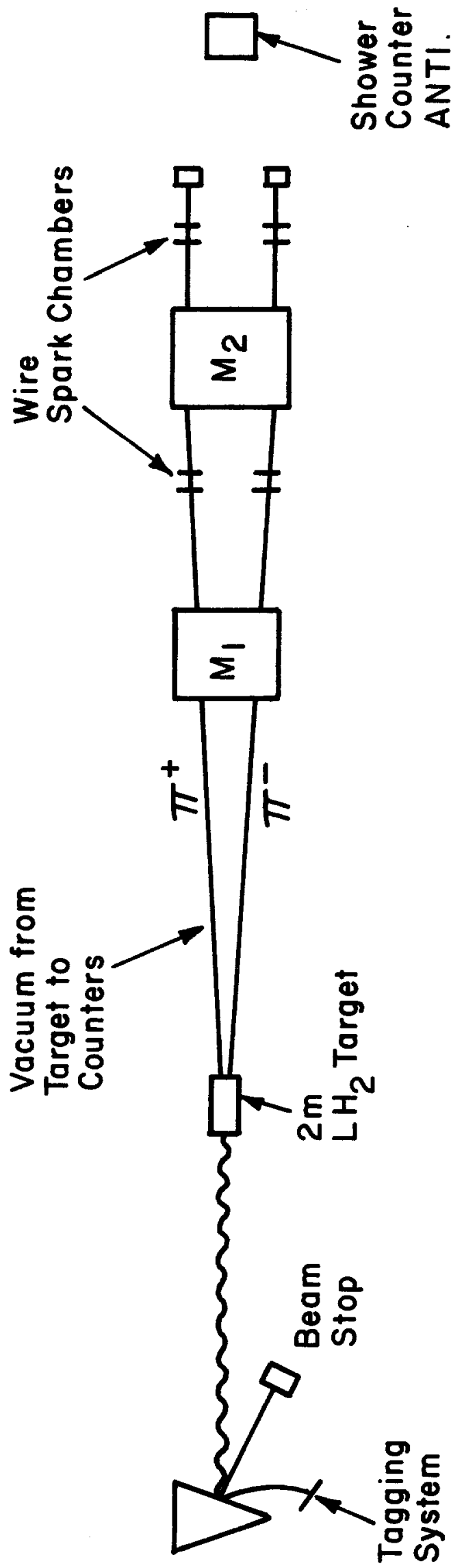

Fig. 4. Vector meson photoproduction experiment. Magnet gap dimensions are $M_{1}=6 \times 1 \times 2 \mathrm{ft}, M_{2}=12 \times 1 \times 2 \mathrm{ft}$, each $18 \mathrm{kG}$. 


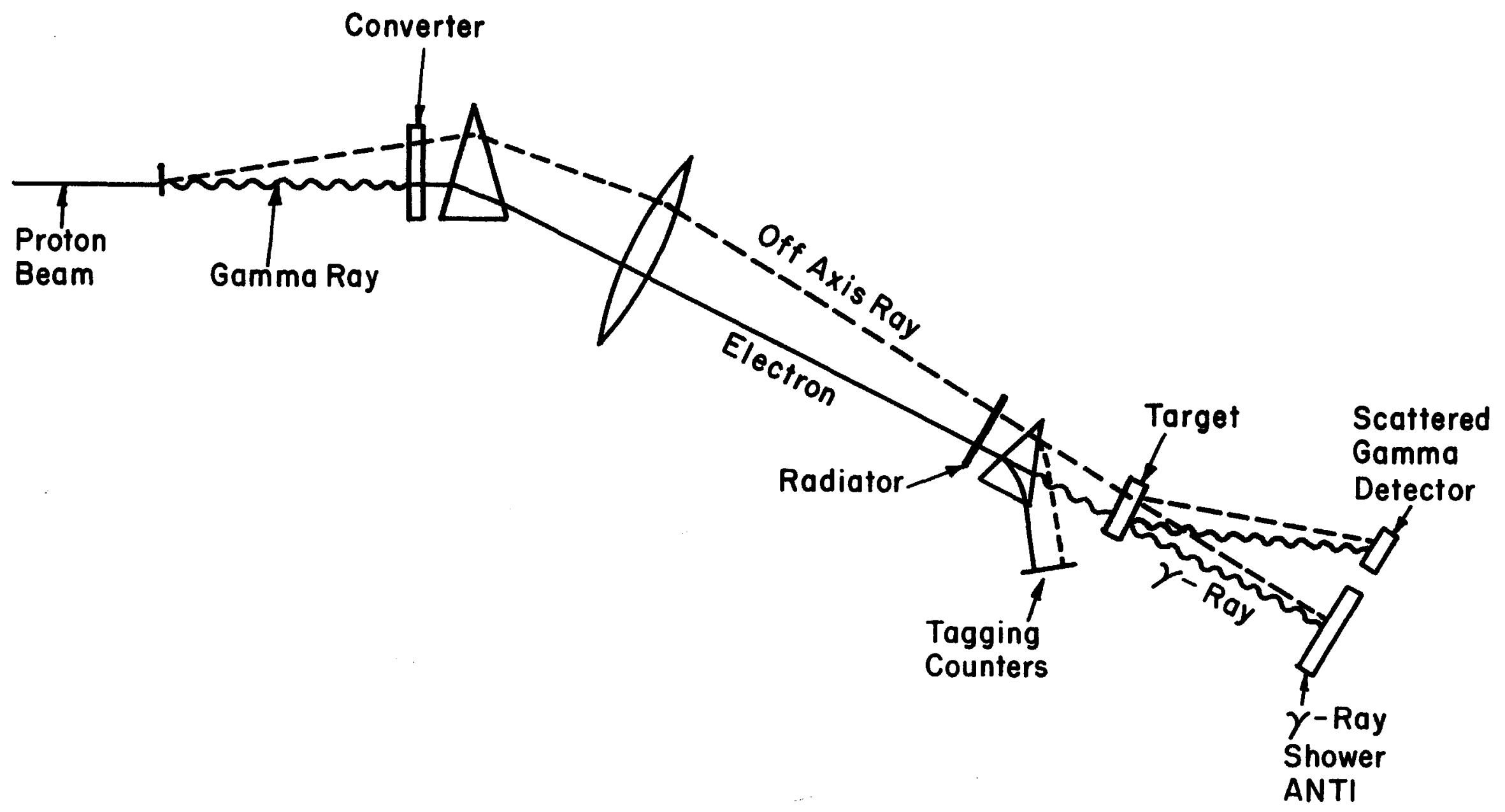

